\title{
A sesquiterpene lactone from Siegesbeckia glabrescens suppresses Hedgehog/Gli-mediated transcription in pancreatic cancer cells
}

\author{
HWA JIN LEE ${ }^{1}$, QIAN WU ${ }^{2}$, HUA LI ${ }^{2}$, GYU-UN BAE ${ }^{2}$, AN KEUN KIM ${ }^{2}$ and JAE-HA RYU ${ }^{2}$ \\ ${ }^{1}$ Department of Natural Medicine Resources, Semyung University, Jecheon, Chungcheongbuk-do 390-711; \\ ${ }^{2}$ Research Center for Cell Fate Control and College of Pharmacy, Sookmyung Women's University, \\ Seoul 140-742, Republic of Korea
}

Received May 7, 2015; Accepted June 16, 2016

DOI: $10.3892 / 01.2016 .4994$

\begin{abstract}
Pancreatic cancer is aggressive and therefore difficult to treat; however, continued efforts have been made with the aim of developing an effective therapy against the disease. The Hedgehog (Hh) signaling pathway is reportedly involved in the proliferation and survival of pancreatic cancer cells. The transcription factor glioma-associated oncogene (Gli) is a key component of the Hh signaling pathway and the primary effector of pancreatic cancer development. Inhibiting Gli is a proven therapeutic strategy for this disease. The present study examined the regulation of Gli and the expression of its target genes to identify an inhibitor of the Sonic Hh (Shh) pathway. A germacranolide sesquiterpene lactone (GSL) was isolated from Siegesbeckia glabrescens as an inhibitor of Gli-mediated transcription. The results demonstrated that GSL inhibited Shh-induced osteoblast differentiation and Gli homolog 1 (Gli1)-mediated transcriptional activity in mesenchymal C3H10T1/2 stem cells. Furthermore, GSL suppressed Gli-mediated transcriptional activity in human pancreatic cancer PANC-1 and AsPC-1 cells, which resulted in reduced cancer cell proliferation and downregulated expression of the Gli-target genes, Gli1 and cyclin D1. A sesquiterpene lactone from $S$. glabrescens may therefore serve as a candidate for the treatment of $\mathrm{Hh} / \mathrm{Gli}$-dependent pancreatic cancer.
\end{abstract}

Correspondence to: Professor Jae-Ha Ryu, Research Center for Cell Fate Control and College of Pharmacy, Sookmyung Women's University, 52 Hyochangwon-Gil, Yongsan-Gu, Seoul 140-742, Republic of Korea

E-mail: ryuha@sookmyung.ac.kr

Abbreviations: Hh, Hedgehog; Shh, Sonic hedgehog; Ptch, 12-pass transmembrane spanning receptor patched; Smo, 7-pass transmembrane receptor Smoothened; Gli, glioma-associated oncogene; Shh CM, Sonic hedgehog conditioned medium

Key words: Siegesbeckia glabrescens, compositae, sesquiterpene lactone, pancreatic cancer, Gli, Hedgehog pathway

\section{Introduction}

Pancreatic cancer is currently one of the leading causes of cancer-associated mortality in industrialized countries, with an incidence rate equaling its mortality rate (1). The number of newly diagnosed cases and mortalities per 100,000 individuals are 12.4 and 10.9 , respectively, according to the National Cancer Institute (Bethesda, MD, USA; http://seer. cancer.gov). Despite research efforts, there has been limited progress regarding the treatment of this disease; therefore, novel strategies to identify new therapeutic agents are urgently required. The Hedgehog $(\mathrm{Hh})$ signaling pathway is critical for morphogenesis signaling and is inappropriately activated in patients with pancreatic cancer $(2,3)$. In addition, this pathway is associated with the growth and metastasis of various types of tumors, including pancreatic cancer $(4,5)$. Therefore, the Hh pathway serves as a therapeutic target, and inhibitors of Hh signaling may function as novel therapeutic drugs for the treatment of pancreatic cancer.

The Hedgehog $(\mathrm{Hh})$ pathway regulates embryonic organogenesis and tissue growth (6), and is activated by $\mathrm{Hh}$ ligands, including Sonic Hh (Shh), which binds to a 12-pass transmembrane spanning receptor known as Patched (Ptch) (7). Binding of the Hh ligand to Ptch relieves repression of a 7-pass transmembrane receptor called Smoothened (Smo) (8). Released Smo activates the signaling pathway, resulting in release of the glioma-associated oncogene (Gli) transcription factor family [including Gli homolog 1 (Gli1), Gli2 and Gli3], which translocate to the nucleus and trigger expression of Gli target genes, such as Gli1 and cyclin D1, for cell growth (9).

Inhibitors of Smo in the Hh pathway have been a frequent focus of therapeutic drug development and have been evaluated in preclinical models $(10,11)$. However, resistance to Smo inhibitors may develop clinically $(12,13)$. Smo inhibitors are ineffective in tumors accompanied by overactivated Gl, downstream of Smo (14). Thus, Gli, which is downstream of Smo, is an important target for repressing activated $\mathrm{Hh}$ signaling. Several studies have reported that Gli1 and Gli2 are the primary transcriptional effectors involved in tumor formation $(15,16)$. In addition, the importance of Gli1 in tumor progression and development is well recognized in human cell culture systems. A number of studies have demonstrated that targeting Gli1 may be a promising cancer therapeutic strategy $(17,18)$. 
During the search for plant-derived inhibitors of the Hh/Gli signaling pathway, the present study observed that a sesquiterpene from Siegesbeckia glabrescens repressed Gli-mediated transcriptional activity and suppressed proliferation of human pancreatic cancer cells. S. glabrescens Makino (Compositae, 'Hi-Chum' in Korea) has been used as an herbal medicine to treat paralysis, inflammatory diseases, allergic disorders and asthma. Extracts of S. glabrescens exhibit antioxidative, antiallergic, anti-inflammatory (19) and anti-tumor activities (20). It was previously reported that a germacranolide sesquiterpene lactone (GSL) from S. glabrescens exhibited anti-inflammatory activity by downregulating inducible nitric oxide synthase and cyclooxygenase-2 expression in lipopolysaccharide-activated macrophages (19). Herein, the present study reports the use of a GSL from $S$. glabrescens as an inhibitor of $\mathrm{Hh} / \mathrm{Gli}$-mediated transcription.

\section{Materials and methods}

Plant material and isolation from S. glabrescens. Part of a S. glabrescens plant (voucher specimen no. SPH 05007 at the College of Pharmacy, Sookmyung Women's University, Seoul, Korea) was obtained from wild at Wan-Do (Jeollanam-do province, Korea) in 2005. The GSL isolation procedure was performed as described previously (19). The GSL structure was confirmed to be 2-propenoic acid, 2-methyl-2,3,3a,4,5, $8,9,10,11,11 a,-d e c a h y d r o-6,10-b i s(h y d r o x y m e t h y l)-3-m e t h y l e n e-$ 2-oxocyclodeca (b) furan-4-yl ester by spectroscopic methods (Fig. 1A).

Cell lines, chemicals and biochemicals. Human pancreatic cancer PANC-1 and AsPC-1 cell lines, and mouse mesenchymal C3H10T1/2 stem cells (American Type Culture Collection, Manassas, VA, USA) were cultured in Dulbecco's modified Eagle's medium (DMEM) containing 10\% fetal bovine serum (FBS) and streptomycin/penicillin (Thermo Fisher Scientific, Inc., Waltham, MA, USA). GANT61 (purity, $\geq 98 \%$ ), a synthetic Gli inhibitor, was purchased from Sigma-Aldrich (St. Louis, MO, USA). Cyclopamine (purity, $\geq 98 \%$; Sigma-Aldrich), a representative plant-derived Smo inhibitor in the Hh pathway, was used as a positive control for the evaluation of the inhibitory properties of the Hh pathway.

Preparation of Shh-conditionedmedia(Shh-CM).Hh signaling was induced by Shh-CM (21). The Shh expression construct was transiently transfected into HEK293 cells to prepare the Shh-CM. Shh-producing HEK293 cells were grown to $80 \%$ confluency in DMEM containing 10\% FBS. The medium was subsequently replaced with DMEM containing 2\% FBS, and allowed to grow for 5 days. The CM was harvested and filtered through a $0.22 \mu \mathrm{m}$ membrane.

Alkaline phosphatase (ALP) assay. C3H10T1/2 cells were seeded at $5 \times 10^{3}$ cells/well in a 96 -well plate and allowed to attach for $4 \mathrm{~h}$, which was followed by the addition of Shh-CM and GSL. Following incubation at $37^{\circ} \mathrm{C}$ for $96 \mathrm{~h}$, $0.9 \% \mathrm{NaCl}$ with $0.2 \%$ Triton $\mathrm{X}-100$ was added to the cells and lysed for $15 \mathrm{~min}$. The cell lysates were mixed with ALP substrate (4 mM p-nitrophenyl phosphate disodium) and reaction buffer $[200 \mathrm{mM}$ Tris- $\mathrm{HCl}(\mathrm{pH} 10.5), 0.4 \mathrm{M}$ 2-amino-2-methylpropanol and $\left.8 \mathrm{mM} \mathrm{MgCl}_{2}\right]$ and incubated in the dark at $37^{\circ} \mathrm{C}$ for $45 \mathrm{~min}$. Absorbance was measured at $415 \mathrm{~nm}$ using a microplate reader (Molecular Devices, LLC, Sunnyvale, CA, USA). The assay was performed in triplicate.

Gli1/Gli-dependent luciferase reporter assay. C3H10T1/2 cells were transiently transfected with Gli1, Gli-dependent firefly luciferase and $\beta$-galactosidase expression constructs (C3H10T1/2-Gli1-Luc cells) to assess Gli1-mediated transcriptional activity. The transfected cells were treated with GSL in the presence of either Shh-CM or HEK293 control medium. Following $30 \mathrm{~h}$ of incubation at $37^{\circ} \mathrm{C}$, Gli1-dependent firefly luciferase and $\beta$-galactosidase activities were measured using a microplate luminometer (Perkin Elmer, Inc., Waltham, MA, USA).

Plasmids of the Gli-dependent firefly luciferase reporter construct and $\beta$-galactosidase expression construct were transiently transfected into PANC-1 cells (PANC-1-Gli-Luc cells) to assess Gli-mediated transcriptional activity in human pancreatic cancer cells. These cells were treated with various concentrations of GSL $(0,1,5,10$ and $20 \mu \mathrm{M})$. Following $20 \mathrm{~h}$ of incubation at $37^{\circ} \mathrm{C}$, cellular firefly luciferase and $\beta$-galactosidase activities were measured with a microplate luminometer.

Cell proliferation assay. Cells were plated at a density of $3 \times 10^{3}$ cells/well in a 96-well plate. The cells were incubated with various concentrations of $\operatorname{GSL}(0,5,10$ and $20 \mu \mathrm{M})$ for 3 days, treated with MTT (5 mg/ml) solution for $4 \mathrm{~h}$, and lysed with dimethyl sulfoxide. Absorbance was read at $540 \mathrm{~nm}$ using a microplate reader. The vehicle-treated group was considered as $100 \%$ of cell proliferation for each cell line.

Western blot analysis. PANC-1 and AsPC-1 cells were treated with various concentrations of $\operatorname{GSL}(0,1,5,10$ and $20 \mu \mathrm{M}$ ) for $20 \mathrm{~h}$, harvested and lysed gently with cell lysis buffer (Cell Signaling Technology, Inc., Danvers, MA, USA). The cell lysates were centrifuged at $10,000 \mathrm{x}$ g for $20 \mathrm{~min}$ at $4^{\circ} \mathrm{C}$ and the supernatants were collected, which were subjected to $10 \%$ sodium dodecyl sulfated-polyacrylamide gel electrophoresis and transferred to polyvinylidene difluoride membranes. Anti-Gli1 (cat. no. 2553), cyclin D1 (cat. no. 2922), phospho-Akt (p-Akt; cat. no. 9271), and total Akt (cat. no. 9272) rabbit polyclonal antibodies (dilution, 1:1,000; Cell Signaling Technology, Inc.) were used for the immunoblot analysis. The bands were detected by LAS3000 (Fujifilm Holdings Corporation, Tokyo, Japan) upon incubation with enhanced chemiluminescence reagents (GE Healthcare Life Sciences, Uppsala, Sweden).

Statistical analysis. Data are presented as the mean \pm standard deviation. P-values were determined with the unpaired Student's $t$-test, which was used to evaluate significant differences between the control and the test group. $\mathrm{P}<0.05$ was considered to indicate a statistically significant difference. All analyses were performed using SPSS 19.0 software (SPSS Inc., Chicago, IL, USA). 


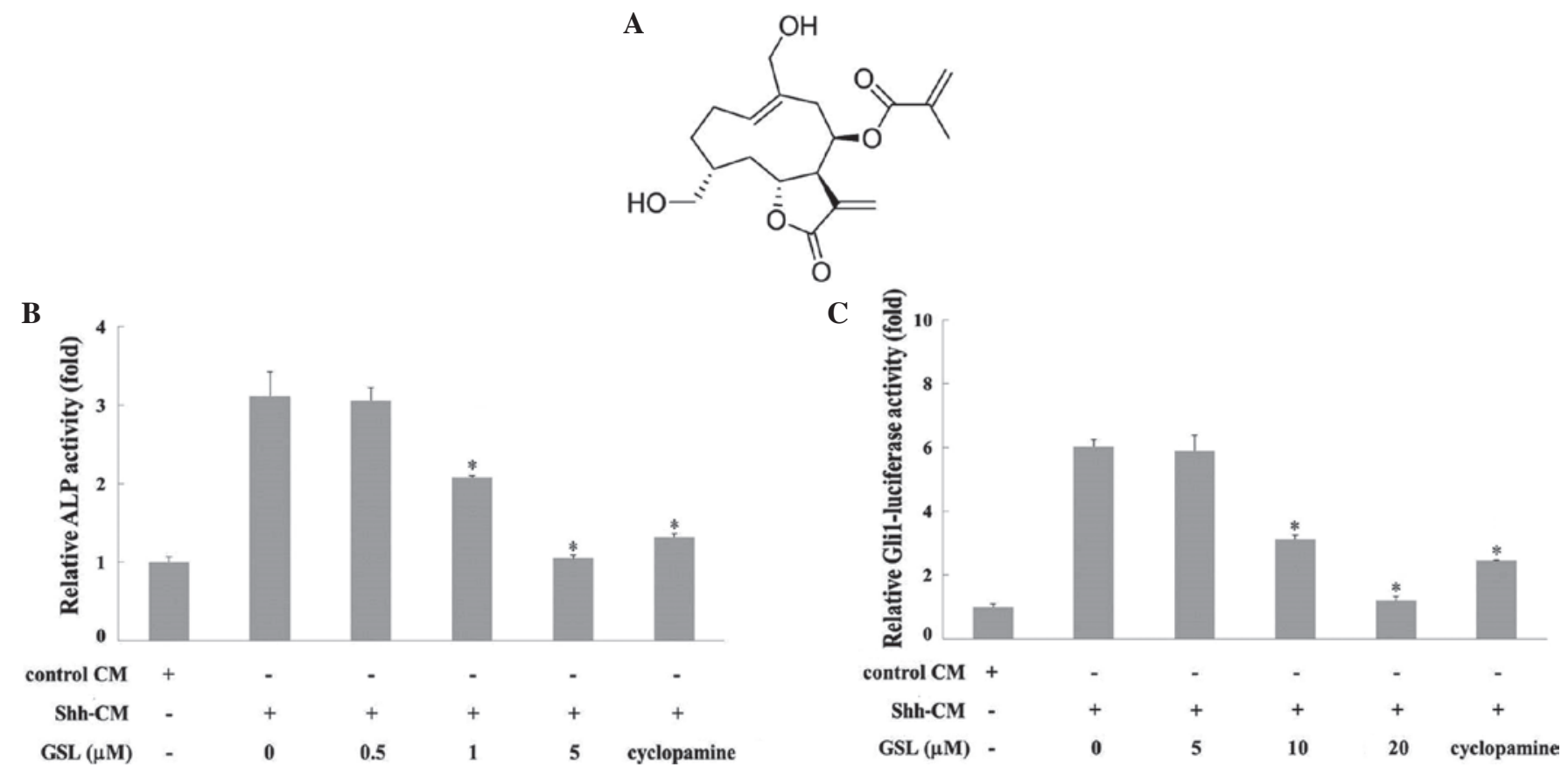

Figure 1. (A) Structure of GSL from Siegesbeckia glabrescens. (B) Effect of GSL on Shh-CM-induced ALP activity. C3H10T1/2 cells were treated with GSL and/or Shh-CM for $96 \mathrm{~h}$ and ALP activity was measured. Cyclopamine $(5 \mu \mathrm{M})$ was used as the positive control. . (C) Effect of GSL on Gli1-mediated transcriptional activity. C3H10T1/2-Gli1-luc cells were seeded onto a 96-well plate and were subsequently induced with Shh-CM. At the same time, various concentrations $(0,5,10$ and $20 \mu \mathrm{M})$ of GSL were added for $30 \mathrm{~h}$. Cyclopamine $(5 \mu \mathrm{M})$ was used as the positive control. The ratio of luciferase reporter activity of the GSL-treated cells/vehicle-treated cells was calculated as the inhibitory effect on Shh-CM-stimulated Gli1-mediated transcriptional activity. "P<0.05 vs. Shh-CM. GSL, germacranolide sesquiterpene lactone; Shh-CM, Sonic Hedgehog conditioned medium; ALP, alkaline phosphatase; Gli1, gliomaassociated oncogene homolog 1 .

\section{Results}

Effects of GSL on ALP activity and Hh/Gli-mediated transcriptional activity in mouse mesenchymal C3H1OTI/2 stem cells. ALP activity was evaluated in Shh-CM-triggered mouse mesenchymal $\mathrm{C} 3 \mathrm{H} 10 \mathrm{~T} 1 / 2$ stem cells to investigate the effects of GSL on the Hh signaling pathway. The $\mathrm{Hh}$ signaling pathway is involved in osteoblast differentiation accompanying activation of ALP as a marker of osteoblast differentiation (22). C3H10T1/2 cells were treated with GSL and/or Shh-CM for 96 h. Cyclopamine, a Smo inhibitor (23), was used as a positive control. As presented in Fig. 1B, GSL decreased ALP activity in Shh-CM-treated C3H10T1/2 cells in a dose-dependent manner compared with the vehicle, which exhibited a high level of ALP activity. These results indicate that GSL inhibits the differentiation of Shh-induced C3H10T1/2 cells into ALP-positive osteoblasts by interfering with Hh signaling. The present study subsequently investigated whether GSL inhibits Shh-induced Gli1-mediated transcription. The activity of Gli1-mediated transcription was measured as luciferase activity in C3H10T1/2-Gli1-Luc cells. Cyclopamine was used as the positive control. As presented in Fig. 1C, GSL treatment inhibited Shh-CM-induced luciferase activity in a dose-dependent manner. $\beta$-galactosidase activities were not affected by GSL up to $20 \mu \mathrm{M}$, indicating no cytotoxic effects. These results suggest that GSL modulates the $\mathrm{Hh} / \mathrm{Gli}$ signaling pathway by suppressing Shh-CM-induced Gli1-mediated transcriptional activity.

Effects of GSL on Gli-mediated transcriptional activity in human pancreatic cancer cells. Gli-mediated transcriptional

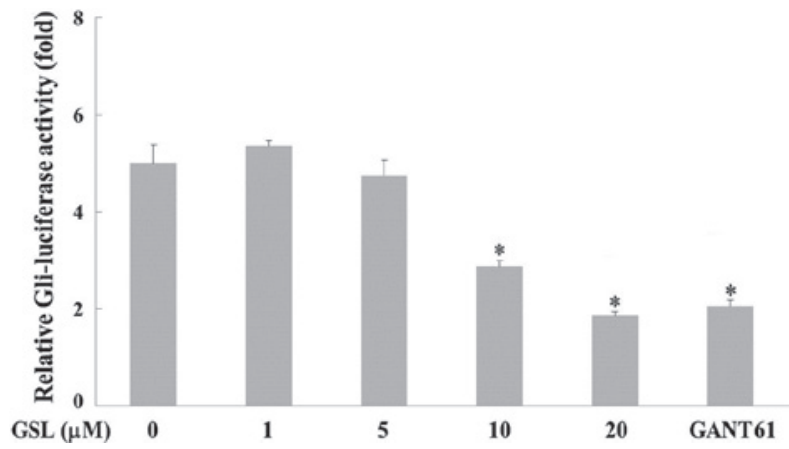

Figure 2. Effect of GSL on Gli-mediated transcriptional activity in human pancreatic cancer PANC-1 cells. PANC-1-Gli-luc cells were seeded onto a 96-well plate and treated with various concentrations $(0,1,5,10$ and $20 \mu \mathrm{M})$ of GSL. GANT61 $(30 \mu \mathrm{M})$ was used as the positive control. Luciferase activity was measured following $20 \mathrm{~h}$ using a microplate luminometer. The ratio of luciferase reporter activity of the GSL-treated cells/vehicle-treated cells was calculated as the inhibitory effect on Gli-mediated transcriptional activity. ${ }^{*} \mathrm{P}<0.05$ vs. control. GSL, germacranolide sesquiterpene lactone; Gli, glioma-associated oncogene.

activity was determined in PANC-1 cells to elucidate the effect of GSL on Shh signaling in human pancreatic cancer. PANC-1 cells, which express aberrantly activated Gli1 (2), were transiently transfected with Gli-dependent firefly and $\beta$-galactosidase reporters (PANC-1-Gli-Luc cells). GSL treatment suppressed luciferase activity in a dose-dependent manner compared with the vehicle treatment, which exhibited high luciferase activity (Fig. 2). GANT61, an inhibitor of Gli-mediated transcription (24), was used as the positive control. The GSL or vehicle treatments did not affect $\beta$-galactosidase 
Table I. Inhibitory effect (\%) of GSL on the proliferation of PANC-1, AsPC-1 and C3H10T1/2 cells.

\begin{tabular}{|c|c|c|c|c|c|}
\hline \multirow[b]{2}{*}{ Cells } & \multicolumn{3}{|c|}{ Concentration of GSL, $\mu \mathrm{M}$} & \multirow[b]{2}{*}{ GANT61 ${ }^{\mathrm{a}}$} & \multirow[b]{2}{*}{$\mathrm{IC}_{50}$ values, $\mu \mathrm{M}$} \\
\hline & 5 & 10 & 20 & & \\
\hline PANC-1 & $48.8 \pm 2.1$ & $62.4 \pm 3.2$ & $70.0 \pm 4.1$ & $82.2 \pm 1.7$ & 6.9 \\
\hline AsPC-1 & $49.9 \pm 0.8$ & $62.1 \pm 2.5$ & $73.1 \pm 2.3$ & $79.7 \pm 3.3$ & 5.1 \\
\hline C3H10T1/2 & $17.0 \pm 1.5$ & $27.8 \pm 1.1$ & $35.3 \pm 2.5$ & $60.8 \pm 2.8$ & 35.7 \\
\hline
\end{tabular}

${ }^{\mathrm{a}} \mathrm{GANT61}(30 \mu \mathrm{M})$ was used as the positive control. GSL, germacranolide sesquiterpene lactone; $\mathrm{IC}_{50}, 50 \%$ inhibitory concentration.

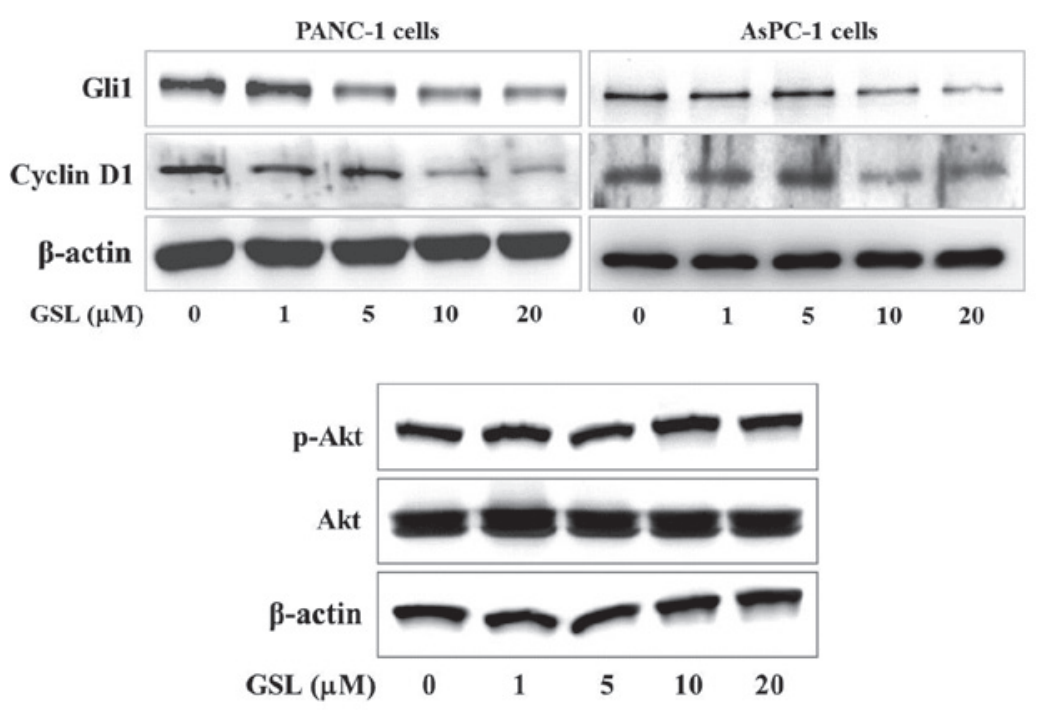

Figure 3. Effect of GSL on Gli1 and cyclin D1 protein levels in human pancreatic cancer cells. Cells were treated with GSL at the indicated concentrations for $20 \mathrm{~h}$. Cell lysates were prepared, and the Gli1, cyclin D1 and $\beta$-actin protein levels were determined by western blotting. Images are representative of three independent experiments with similar results. GSL, germacranolide sesquiterpene lactone; Gli1, glioma-associated oncogene homolog 1; p-, phospho.

activities, indicating that GSL inhibits Gli-mediated transcriptional activity in human pancreatic cancer cells.

Effects of GSL on the proliferation of human pancreatic cancer cells. Next, the current study investigated whether GSL affects Gli-mediated proliferation in human pancreatic cells (PANC-1 and AsPC-1 cells) with an increased Hh signal. C3H10T1/2 cells were used as controls with no dependence on the Hh signal. GANT61 was used the positive control Gli-inhibitor that inhibits cell proliferation. As presented in Table I, GSL dose-dependently suppressed proliferation of the pancreatic cancer cells, with $50 \%$ inhibitory concentration $\left(\mathrm{IC}_{50}\right)$ values of 6.9 and $5.1 \mu \mathrm{M}$ in PANC-1 and AsPC-1 cells, respectively. However, GSL exhibited a weak inhibitory effect on the proliferation of C3H10T1/2 control cells, with an $\mathrm{IC}_{50}$ value of $35.7 \mu \mathrm{M}$.

Effects of GSL on Gli-related protein levels in human pancreatic cancer cells. The present study examined the effect of GSL on Gli-mediated protein expression, including Gli1 and cyclin D1, as key regulators of tumor cell growth. Immunoblot analysis demonstrated that GSL reduced the expression of Gli1 and cyclin D1 proteins in a concentration-dependent manner compared with that of the vehicle treated PANC-1 and AsPC-1 cells (Fig. 3). It has been previously reported that phosphatidylinositol 3-kinase (PI3K)/Akt signaling interferes with Gli signaling without inhibiting Smo (25). Thus, the current study examined the effect of GSL on PI3K/Akt signaling, and it was observed that GSL had no effect on p-Akt/total Akt levels in the PANC-1 cells (Fig. 3). These results indicate that GSL suppressed proliferation of pancreatic cancer cells by downregulating Gli1 and cyclin D1 expression through repression of Gli-mediated transcription and did not disrupt PI3K/Akt signaling.

\section{Discussion}

The Hh pathway is responsible for regulating and coordinating cellular growth and development in the embryo, and is implicated in the formation and development of tumors, including pancreatic cancer $(26,27)$. The $\mathrm{Hh}$ pathway is activated by binding of Shh to Ptch, which activates Smo and subsequently the Gli family (28). The Gli family has three mammalian homologs; Gli1 and Gli2 function as activators of Hh signaling to induce the expression of target genes, including Gli1 and cyclin D, whilst Gli3 is a repressor of $\mathrm{Hh}$ signaling $(29,30)$. Gli1 and Gli2 regulate the expression of target genes related to cell growth and survival; however, Gli2 may function as an effective transcription factor in the absence of Gli1 (31). 
Recent evidence suggests a critical role of Hh signaling in tumorigenesis through mutations in Ptch or Smo, which lead to hyperactivation of Gli in certain tumor types, including pancreatic cancer (32). Constitutive activation of Gli is associated with tumor formation and growth (33-35). Although Smo antagonists have been studied to treat Hh-directed cancer (36), therapeutic efficiencies were extremely low in preclinical and clinical models of tumors accompanying activation of Smo-downstream components, including Gli $(37,38)$. Therefore, targeting the Hh pathway at the level of Gli rather than at the level of Smo may be a better strategy for treating cancer associated with an uncontrolled Hh pathway.

In the present study, it was observed that GSL from S. glabrescens inhibited the $\mathrm{Hh} / \mathrm{Gli}$ signaling pathway in C3H10T1/2 cells (Fig. 1). Mesenchymal stem cells differentiate into osteoblasts following $\mathrm{Hh}$ signal activation, and high ALP activity may be measured as a marker of osteoblast differentiation (39). Mesenchymal C3H10T1/2 stem cells were treated with Shh-CM to induce osteoblast differentiation through activation of the Hh pathway. GSL inhibited Shh-induced ALP activity in the C3H10T1/2 cells by suppressing the $\mathrm{Hh}$ signaling pathway. Luciferase activity was measured in C3H10T1/2-Gli1-Luc cells to evaluate the inhibitory potential of GSL on Shh-induced Gli-mediated transcription. While Shh-CM increased luciferase activity by inducing Gli1 transcription, GSL inhibited luciferase activity in a dose-dependent manner. These results indicate that GSL inhibits the Hh/Gli signaling pathway, consistent with the ALP activity result.

The majority of inhibitors of the Hh signaling pathway target Smo; however, other mechanisms of $\mathrm{Hh}$ signal-directed cancer have been reported, including Ptch mutations or overexpression of the Shh ligand or Gli in pancreatic cancer $(40,41)$. The present study examined the inhibitory potential of GSL on Gli-mediated transcription in human pancreatic cancer PANC-1 cells, which overexpress Gli and are Smo insensitive $(2,42)$. The inhibition of Gli-mediated transcription activity suggests that GSL suppresses downstream of Gli to inhibit cancer cell proliferation or gene expression.

Furthermore, the current study observed that GLS suppressed Gli-mediated proliferation of the human pancreatic cancer PANC-1 and AsPC-1 cells. PANC-1 cells are reported to be cyclopamine-insensitive (42), whereas AsPC-1 cells are cyclopamine-sensitive and $\mathrm{Hh}$ signaling-dependent cancer cells, as they overproduce Shh (43). As presented in Table I, the anti-proliferative potential of GSL against pancreatic cancer cells is stronger than that against the control C3H10T1/2 cells.

Several plant-derived modulators of the $\mathrm{Hh} / \mathrm{Gli}$ signaling pathway have been reported, including cyclopamine (steroidal alkaloid) as a Smo antagonist, and staurosporinone (bisindole alkaloid) and zerumbone (sesquiterpene) as inhibitors of Gli-mediated transcription (44). Hosoya et al (44) reported that the $\alpha, \beta$-unsaturated carbonyl group in zerumbone is important for inhibiting Gli-mediated transcription, but zerumbone has similar cytotoxicity in PANC-1 and C3H10T1/2 cells. In the present study, GSL was 5-fold more toxic against the PANC-1 and AsPC-1 cells than it was against the $\mathrm{C} 3 \mathrm{H} 10 \mathrm{~T} 1 / 2$ cells. The structural requirements for cell-type selective toxicity of GSL in $\mathrm{Hh} / \mathrm{Gli}$ signaling requires clarification in further investigations.
Gli1 and cyclin D1 expression is dependent on Gli-mediated transcription (45). Gli1, a target gene of the Gli transcription factor, regulates transcription of the Hh responsive genes by itself (46). Gli1 is upregulated in the majority of pancreatic cancer tissues and its level of expression is positively correlated with $\mathrm{Hh}$ signaling (47). Cyclin D1, another Gli target gene, functions as a cell cycle regulator and is important in carcinogenesis $(48,49)$. In the present study, basal levels of Gli1 and cyclin D1 were elevated in PANC-1 and AsPC-1 cells. GSL downregulated Gli1 and cyclin D1 protein levels in a dose-dependent manner (Fig. 3). These results are in accordance with the decline of Gli-mediated transcriptional activity and proliferation of pancreatic cancer cells. Furthermore, $\mathrm{p}$-Akt/total Akt protein levels were not affected by GSL treatment in the PANC-1 cells (Fig. 3), suggesting that GSL has no effect on the PI3K/Akt signaling pathway, which may be involved specifically in regulating $\mathrm{Hh} / \mathrm{Gli}$ signaling.

In conclusion, the current study identified a GSL derived from $S$.glabrescens that functions as a modulator of the $\mathrm{Hh} / \mathrm{Gli}$ signaling pathway. This compound exerted anti-proliferative effects against human pancreatic cancer PANC-1 and AsPC-1 cells, at least partially, by downregulating Gli-mediated transcriptional activity, and Gli1 and cyclin D1 expression. GSL from $S$. glabrescens may be a valuable candidate for the development of novel therapeutic agents against $\mathrm{Hh} / \mathrm{Gli}$-dependent forms of cancer.

\section{Acknowledgements}

The present study was supported by grants from the National Research Foundation of Korea funded by the Korean Government (MSIP) (nos. 2011-0030074 and 2012R1A1A3013645).

\section{References}

1. Bosetti C, Bertuccio P, Negri E, La Vecchia C, Zeegers MP and Boffetta P. Pancreatic cancer: Overview of descriptive epidemiology. Mol Carcinog 51: 3-13, 2012.

2. Guo J, Gao J, Li Z, Gong Y, Man X, Jin J and Wu H: Adenovirus vector-mediated Gli1 siRNA induces growth inhibition and apoptosis in human pancreatic cancer with Smo-dependent or Smo-independent Hh pathway activation in vitro and in vivo. Cancer Lett 339: 185-194, 2013.

3. Hao K, Tian XD, Qin CF, Xie XH and Yang YM: Hedgehog signaling pathway regulates human pancreatic cancer cell proliferation and metastasis. Oncol Rep 29: 1124-1132, 2013.

4. Morton JP, Mongeau ME, Klimstra DS, Morris JP, Lee YC, Kawaguchi Y, Wright CV, Hebrok M and Lewis BC: Sonic hedgehog acts at multiple stages during pancreatic tumorigenesis. Proc Natl Acad Sci USA 104: 5103-5108, 2007.

5. Kelleher FC: Hedgehog signaling and therapeutics in pancreatic cancer. Carcinogenesis 32: 445-451, 2011.

6. Ingham PW and McMahon AP: Hedgehog signaling in animal development: Paradigms and principles. Genes Dev 15: 3059-3087, 2001.

7. Stone DM, Hynes M, Armanini M, Swanson TA, Gu Q Johnson RL, Scott MP, Pennica D, Goddard A, Phillips H, et al: The tumour-suppressor gene patched encodes a candidate receptor for Sonic hedgehog. Nature 384: 129-134, 1996.

8. Akiyama H, Shigeno C, Hiraki Y, Shukunami C, Kohno H, Akagi M, Konishi J and Nakamura T: Cloning of a mouse smoothened cDNA and expression patterns of hedgehog signalling molecules during chondrogenesis and cartilage differentiation in clonal mouse EC cells, ATDC5. Biochem Biophys Res Commun 235: 142-147, 1997

9. Kasper M, Regl G, Frischauf AM and Aberger F: GLI transcription factors: Mediators of oncogenic hedgehog signaling. Eur J Cancer 42: 437-445, 2006. 
10. Ailles L and Siu LL: Targeting the Hedgehog pathway in cancer: Can the spines be smoothened? Clin Cancer Res 17: 2071-2073, 2011.

11. Tang JY and Marghoob AA: Emerging treatments and signaling pathway inhibitors. Semin Cutan Med Surg 30 (Suppl 4): S14-S18, 2011.

12. Schnidar H, Eberl M, Klingler S, Mangelberger D, Kasper M, Hauser-Kronberger C, Regl G, Kroismayr R, Moriggl R, Sibilia M and Aberger F: Epidermal growth factor receptor signaling synergizes with hedgehog/GLI in oncogenic transformation via activation of the MEK/ERK/JUN pathway. Cancer Res 69: 1284-1292, 2009.

13. LoRusso PM, Rudin CM, Reddy JC, Tibes R, Weiss GJ, Borad MJ, Hann CL, Brahmer JR, Chang I, Darbonne WC, et al: Phase I trial of hedgehog pathway inhibitor vismodegib (GDC-0449) in patients with refractory, locally advanced or metastatic solid tumors. Clin Cancer Res 17: 2502-2511, 2011.

14. Mazumdar T, Devecchio J, Agyeman A, Shi $\mathrm{T}$ and Houghton JA: Blocking Hedgehog survival signaling at the level of the GLI genes induces DNA damage and extensive cell death in human colon carcinoma cells. Cancer Res 71: 5904-5914, 2011.

15. Kimura H, Stephen D, Joyner A and Curran T: Gli1 is important for medulloblastoma formation in Ptc1+/- mice. Oncogene 24: 4026-4036, 2005.

16. Thiyagarajan S, Bhatia N, Reagan-Shaw S, Cozma D, ThomasTikhonenko A, Ahmad N and Spiegelman VS: Role of GLI2 transcription factor in growth and tumorigenicity of prostate cells. Cancer Res 67: 10642-10646, 2007.

17. Lauth M, Bergström A, Shimokawa T and Toftgård R: Inhibition of GLI-mediated transcription and tumor cell growth by small-molecule antagonists. Proc Natl Acad Sci USA 104: 8455-8460, 2007.

18. Zahreddine HA, Culjkovic-Kraljacic B, Assouline S, Gendron P, Romeo AA, Morris SJ, Cormack G, Jaquith JB, Cerchietti L, Cocolakis E, et al: The sonic hedgehog factor GLI1 imparts drug resistance through inducible glucuronidation. Nature 511: 90-93, 2014.

19. Li H, Kim JY, Hyeon J, Lee HJ and Ryu JH: In vitro antiinflammatory activity of a new sesquiterpene lactone isolated from Siegesbeckia glabrescens. Phytother Res 25: 1323-1327, 2011.

20. Cho YR, Choi SW and Seo DW: The in vitro antitumor activity of Siegesbekia glabrescens against ovarian cancer through suppression of receptor tyrosine kinase expression and the signaling pathways. Oncol Rep 30: 221-226, 2013.

21. Chen JK, Taipale J, Young KE, Maiti T and Beachy PA: Small molecule modulation of Smoothened activity. Proc Natl Acad Sci USA 99: 14071-14076, 2002.

22. Nakamura TT, Aikawa M, Iwamoto-Enomoto M, Iwamoto M, Higuchi Y, Pacifici M, Kinto N, Yamaguchi A, Noji S, Kurisu K and Matsuya T: Induction of osteogenic differentiation by hedgehog proteins. Biochem Biophys Res Commun 237: 465-469, 1997

23. Lee ST, Welch KD, Panter KE, Gardner DR, Garrossian M and Chang CW: Cyclopamine: From cyclops lambs to cancer treatment. J Agric Food Chem 62: 7355-7362, 2014.

24. Stanton BZ and Peng LF: Small-molecule modulators of the Sonic hedgehog signaling pathway. Mol Biosyst 6: 44-54, 2009.

25. Riobó NA, Lu K, Ai X, Haines GM and Emerson CP Jr: Phosphoinositide 3-kinase and akt are essential for Sonic hedgehog signaling. Proc Natl Acad Sci USA 103: 4505-4510, 2006.

26. Lauth $M$ and Toftgård R: Hedgehog signaling and pancreatic tumor development. Adv Cancer Res 110: 1-17, 2011.

27. Xie J, Bartels CM, Barton SW and Gu D: Targeting hedgehog signaling in cancer: Research and clinical developments. Onco Targets Ther 6: 1425-1435, 2013.

28. Park HL, Bai C, Platt KA, Matise MP, Beeghly A, Hui CC, Nakashima M and Joyner AL: Mouse Gli1 mutants are viable but have defects in SHH signaling in combination with a Gli2 mutation. Development 127: 1593-1605, 2000.

29. Katoh Y and Katoh M: Hedgehog signaling pathway and gastrointestinal stem cell signaling network (review). Int J Mol Med 18: 1019-1023, 2006.
30. Hu MC, Mo R, Bhella S, Wilson CW, Chuang PT, Hui CC and Rosenblum ND: GLI3-dependent transcriptional repression of Gli1, Gli2 and kidney patterning genes disrupts renal morphogenesis. Development 133: 569-578, 2006.

31. Bai CB and Joyner AL: Gli1 can rescue the in vivo function of Gli2. Development 128: 5161-5172, 2001.

32. Di Magno L, Coni S, Di Marcotullio L and Canettieri G: Digging a hole under Hedgehog: Downstream inhibition as an emerging anticancer strategy. Biochim Biophys Acta 1856: 62-72, 2015.

33. Reifenberger J, Wolter M, Knobbe CB, Köhler B, Schönicke A, Scharwächter C, Kumar K, Blaschke B, Ruzicka T and Reifenberger G: Somatic mutations in the PTCH, SMOH, SUFUH and TP53 genes in sporadic basal cell carcinomas. Br J Dermatol 152: 43-51, 2005.

34. Bahra M, Kamphues C, Boas-Knoop S, Lippert S, Esendik U, Schüller U, Hartmann W, Waha A, Neuhaus P, Heppner F, et al: Combination of hedgehog signaling blockage and chemotherapy leads to tumor reduction in pancreatic adenocarcinomas. Pancreas 41: 222-229, 2012.

35. Wang XD, Inzunza H, Chang H, Qi Z, Hu B, Malone D and Cogswell J: Mutations in the hedgehog pathway genes SMO and PTCH1 in human gastric tumors. PLoS One 8: e54415, 2013.

36. Bisht S, Brossart P, Maitra A and Feldmann G: Agents targeting the hedgehog pathway for pancreatic cancer treatment. Curr Opin Investig Drugs 11: 1387-1398, 2010.

37. Lauth $\mathrm{M}$ and Toftgård R: Non-canonical activation of GLI transcription factors: Implications for targeted anti-cancertherapy. Cell Cycle 6: 2458-2463, 2007.

38. Lee Y, Kawagoe R, Sasai K, Li Y, Russell HR, Curran T and McKinnon PJ: Loss of suppressor-of-fused function promotes tumorigenesis. Oncogene 26: 6442-6447, 2007.

39. Kim WK, Meliton V, Bourquard N, Hahn TJ and Parhami F: Hedgehog signaling and osteogenic differentiation in multipotent bone marrow stromal cells are inhibited by oxidative stress. J Cell Biochem 111: 1199-1209, 2010.

40. Fendrich V, Oh E, Bang S, Karikari C, Ottenhof N, Bisht S, Lauth M, Brossart P, Katsanis N, Maitra A and Feldmann G. Ectopic overexpression of Sonic Hedgehog (Shh) induces stromal expansion and metaplasia in the adult murine pancreas. Neoplasia 13: 923-930, 2011.

41. Bahra M, Kamphues C, Boas-Knoop S, Lippert S, Esendik U, Schüller U, Hartmann W, Waha A, Neuhaus P, Heppner F, et al: Combination of hedgehog signaling blockage and chemotherapy leads to tumor reduction in pancreatic adenocarcinomas. Pancreas 41: 222-229, 2012.

42. Thayer SP, di Magliano MP, Heiser PW, Nielsen CM, Roberts DJ, Lauwers GY, Qi YP, Gysin S, Fernández-del Castillo C, Yajnik V, et al: Hedgehog is an early and late mediator of pancreatic cancer tumorigenesis. Nature 425: 851-856, 2003.

43. Hu WG, Liu T, Xiong JX and Wang CY: Blockade of sonic hedgehog signal pathway enhances antiproliferative effect of EGFR inhibitor in pancreatic cancer cells. Acta Pharmacol Sin 28: 1224-1230, 2007.

44. Hosoya T, Arai MA, Koyano T, Kowithayakorn T and Ishibashi M: Naturally occurring small-molecule inhibitors of hedgehog/GLI-mediated transcription. Chembiochem 9: 1082-1092, 2008.

45. Hyman JM, Firestone AJ, Heine VM, Zhao Y, Ocasio CA, Han K, Sun M, Rack PG, Sinha S, Wu JJ, et al: Small-molecule inhibitors reveal multiple strategies for hedgehog pathway blockade. Proc Natl Acad Sci USA 106: 14132-14137, 2009.

46. Katoh Y and Katoh M. Hedgehog target genes: Mechanisms of carcinogenesis induced by aberrant hedgehog signaling activation. Curr Mol Med 9: 873-886, 2009.

47. Xu X, Su B, Xie C, Wei S, Zhou Y, Liu H, Dai W, Cheng P, Wang F, Xu X and Guo C: Sonic hedgehog-Glil signaling pathway regulates the epithelial mesenchymal transition (EMT) by mediating a new target gene, S100A4, in pancreatic cancer cells. PLoS One 9: e9644, 2014.

48. Gill PS and Rosenblum ND: Control of murine kidney development by sonic hedgehog and its GLI effectors. Cell Cycle 5: 1426-1430, 2006.

49. Onishi $\mathrm{H}$ and Katano M: Hedgehog signaling pathway as a new therapeutic target in pancreatic cancer. World J Gastroenterol 20: 2335-2342, 2014. 